significantly higher in SE $(84.51 \%$ vs $81.57 \%$, p < 0.01). However, rates of Pseudomonas aenginosa, Burkholderia cepacia and MRSA were similar. Significantly higher proportions of patients were diagnosed before turning 3 months in the NE compared to SE (46.76\% vs $42.43 \%)$. In adults: in the NE the BMI was higher 22.35 vs 21.99 ( $\mathrm{p}<0.01$ ) as was the FEV1\%p, adjusted for age and sex $(66.73 \%$ vs $64.69 \%, \mathrm{p}=0.04)$. Patients in SE were more frequently prescribed mucolytics (Dornase Alfa and Hypertonic Saline). In NE they more frequently used chronic macrolides. There were higher rates of PA, Bcc and MRSA in NE. The rates of MSSA and NTM were higher in the SE.

Conclusions There is a north-south divide in demographic characteristics and clinical outcomes in cystic fibrosis (CF) patients in England. In SE children have higher lung function. However, adults in the NE seem to have higher lung function compared to adults in SE. A single year cohort is not sufficient to deduce if these differences affect longer-term outcomes, like survival and requires further investigation.

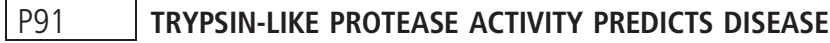 SEVERITY AND PATIENT MORTALITY IN ADULTS WITH CYSTIC FIBROSIS}

${ }^{1} \mathrm{JA}$ Reihill, ${ }^{1} \mathrm{KL}$ Moffitt, ${ }^{2} \mathrm{AM}$ Jones, ${ }^{1} \mathrm{JS}$ Elborn, 'SL Martin. 'Queen's University Belfast, Belfast, UK; ${ }^{2}$ Manchester Adult Cystic Fibrosis Centre, Manchester, UK

\subsection{6/thoraxjnl-2016-209333.234}

Introduction Serine trypsin-like (TL) proteases, which are excessively active in CF airways, promote activation of the epithelial sodium channel $(\mathrm{ENaC})$ and airways dehydration; a key initiating factor for CF lung disease pathogenesis. Furthermore TL-proteases enhance mucin gene expression and mucus hypersecretion, yet whether there is any relationship between the activity of these enzymes and CF pulmonary disease is unknown.

Objectives The primary objective of the current investigation was to determine whether TL-protease activity, measured in adult CF sputum sol, correlates with lung disease and patient outcome (survival). A secondary objective was to compare the strength of any relationships observed with that of neutrophil elastase (NE), an established protease biomarker.

Methods In this cross sectional retrospective study we analysed CF sputum sol collected from 30 clinically stable adult CF patients. Protease activity was measured by monitoring the hydrolysis of peptide-based substrates. Biomarkers of inflammation (IL- 8 and TNF- $\alpha$ ) were measured by ELISA. Lung function was assessed by spirometry (FEV1). Mortality data was retrospectively obtained and time in months until death or transplantation used for subsequent survival analysis.

Results TL-protease activity inversely correlated with lung function (FEV1) $(\mathrm{r}=-0.4, \mathrm{p}=0.031)$ however, no relationship with IL-8 and TNF $\alpha$ was observed. In contrast, NE was found to correlate with IL-8: $\mathrm{r}=0.7, \mathrm{p}<0.001$ and TNF $\alpha$ : $\mathrm{r}=0.7$, $\mathrm{p}<0.001$ but showed no relationship with lung function, indicating that these serine proteases play very distinct roles within the disease process. Kaplan-Meier analysis demonstrated significantly reduced survival for those individuals with above median TL-protease activity. Levels of NE activity showed no relationship with patient survival. Using a multivariate Cox regression analysis (adjusted for age and BMI) a significantly increased mortality hazard (HR 1.028, 95\% CI: 1.007-1.049; p = 0.009) was also identified. These findings are supported by analysis of a validation cohort consisting of samples collected from a separate cohort of 33 adult CF patients.

Conclusions TL-protease activity inversely correlates with lung function and patient survival. As such tryptic activity may warrant consideration when modelling CF survivorship and should be investigated further as a biomarker of CF lung disease and as a potential therapeutic target.

\section{P92 SYSTEMIC ALKYL QUINOLONES AS NOVEL BIOMARKERS FOR PULMONARY EXACERBATIONS IN CYSTIC FIBROSIS: A VALIDATION STUDY}

${ }^{1} \mathrm{H}$ Barr, ${ }^{1} \mathrm{~A}$ Fogarty, ${ }^{1} \mathrm{~N}$ Halliday, ${ }^{1} \mathrm{~A}$ Knox, ${ }^{2} \mathrm{~B}$ Quon, ${ }^{1} \mathrm{D}$ Forrester, ${ }^{1} \mathrm{P}$ Williams, ${ }^{1} \mathrm{D}$ Barrett, ${ }^{1} \mathrm{M}$ Camara. ${ }^{1}$ University of Nottingham, Nottingham, UK; ${ }^{2}$ University of British Columbia, Vancouver, Canada

\subsection{6/thoraxjnl-2016-209333.235}

Introduction and objectives There is a clinical need to identify and validate biomarkers that are sensitive to treatment of infection in cystic fibrosis (CF). The aim of this study was to externally validate two novel biomarkers for pulmonary exacerbations in $\mathrm{CF}$ of the alkyl quinolone (AQ) class of quorum sensing molecules produced by Pseudomonas aeruginosa.

Methods Retrospective analysis of 70 plasma samples from thirteen adults with CF obtained during treatment of fifteen discrete exacerbations treated with intravenous antibiotics. Plasma samples were obtained at the start, day five, day ten, at the end of treatment, and at clinical stability. Samples were analysed using liquid chromatography-mass spectrometry. Data were analysed using Spearman's rank correlations and Wilcoxon matched pairs signed-rank tests using STATA 11 statistical software (Texas, USA). Graphs were produced in EXCEL 2011.

Results Plasma 2-heptyl-4-hydroxyquinoline (HHQ) concentration significantly decreased by a median of $221 \mathrm{pmol} / \mathrm{L}$ (IQR: 158 to $258 \mathrm{pmol} / \mathrm{L}$ ) or $73 \%$ (IQR 52 to $94 \%$; p $=0.0007$ ) during treatment for a pulmonary exacerbation (Figure 1). In the same interval, there was no significant change in plasma NHQ (median decrease of $-3 \mathrm{pmol} / \mathrm{L}$; IQR: -35 to $10 \mathrm{pmol} / \mathrm{L}$; $\mathrm{p}=0.65)$. During treatment for a pulmonary exacerbation, percent predicted FEV1 increased by $4 \%$ (IQR: 1 to $7 \%$; $\mathrm{p}=0.0086$ ). Folloing systemic antimicrobial therapy, systemic IL6 concentration decreased by a median of $2.06 \mathrm{pg} / \mathrm{mL}$ (IQR: 1.02 to $3.55 \mathrm{pg} / \mathrm{mL} ; \mathrm{p}=0.0022$ ) and systemic calprotectin

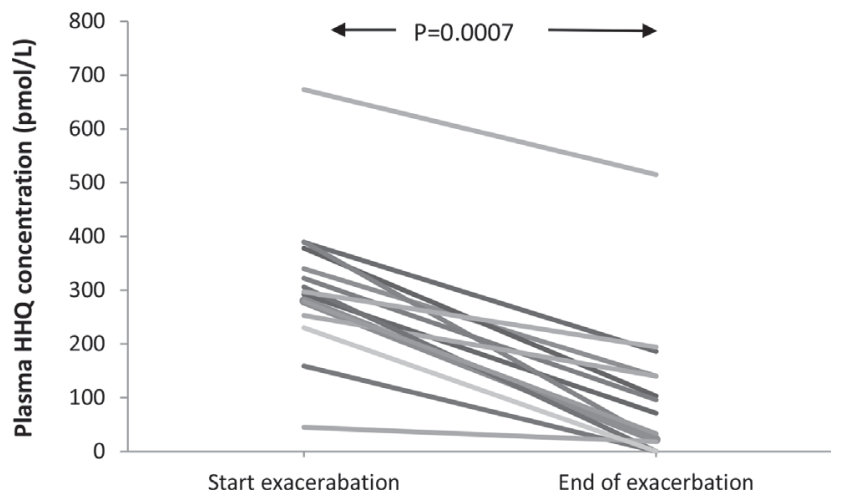

Abstract P92 Figure 1 Plasma HHQ concentrations at the start and end of 15 pulmonary exacerbations treated with systemic antipseudomonal antibiotics in 13 adults with CF

HHQ = 2-heptyl-4-hydroxyquinoline

$\mathrm{pmol} / \mathrm{L}=$ picomoles per litre 